\title{
Functional domains on elastin and microfibril-associated glycoprotein involved in elastic fibre assembly
}

\author{
Patricia BROWN-AUGSBURGER ${ }^{\star}$, Thomas BROEKELMANN${ }^{\star} \uparrow$, Joel ROSENBLOOM $\ddagger$ and Robert P. MECHAM ${ }^{\star} \uparrow \S$ \\ *Department of Cell Biology and Physiology and †Department of Medicine, Washington University School of Medicine, Box 8228, 660 South Euclid Avenue, St. Louis, \\ M0 63110, U.S.A., and \$Department of Anatomy and Histology, School of Dental Medicine, University of Pennsylvania, 4001 Spruce Street, Philadelphia, PA 19104, \\ U.S.A.
}

Studies in vitro suggest that the C-terminus of tropoelastin mediates elastin polymerization through an interaction with microfibril-associated proteins. In this study we have used cultured auricular chondrocytes as a model system to examine whether this interaction is critical for elastic fibre formation in vivo. Auricular chondrocytes, which deposit an abundant elastic fibre matrix, were cultured in the presence of Fab fragments of antibodies directed against the $\mathrm{C}$-terminus $\left(\mathrm{CT}_{\mathrm{e}}\right)$ or an $\mathrm{N}$-terminal domain $\left(\mathrm{AT}_{\mathrm{e}}\right)$ of tropoelastin. Immunofluorescent staining of the extracellular matrix deposited by the cells showed that the $\mathrm{CT}_{\mathrm{e}}$ antibody inhibited the deposition of elastin without affecting microfibril structure. Cells grown under identical conditions in the presence of $\mathrm{AT}_{\mathrm{e}}$, however, formed fibres that stained normally for both elastin and microfibril proteins. Chondrocytes cultured in the presence of microfibril-associated glycoprotein (MAGP):21-35, an antibody directed against a domain near the
$\mathrm{N}$-terminus of MAGP, did not organize tropoelastin into fibres. However, immunostaining for MAGP and fibrillin revealed normal microfibrils. In agreement with the immunofluorescence staining patterns, fewer elastin-specific cross-links, indicative of insoluble elastin, were detected in the extracellular matrix of cells cultured in the presence of $\mathrm{CT}_{\mathrm{e}}$. The medium from these cultures, however, contained more soluble elastin, consistent with an antibody-induced alteration of elastin assembly but not its synthesis. Northern analysis of antibody-treated and control cultures substantiated equivalent levels of tropoelastin mRNA. These results confirm that the C-terminus of tropoelastin interacts with microfibrils during the assembly of elastic fibres. Further, the results suggest that the interaction between tropoelastin and microfibrils might be mediated by a domain involving the $\mathrm{N}$ terminal half of MAGP.

\section{INTRODUCTION}

Elastic fibres provide mechanical recoil from stretching forces in tissues such as lung, aorta, ligament and skin. The main component of elastic fibres is the protein elastin, which provides the characteristic recoil properties. Like rubber, which derives its recoil properties in part from its polymeric nature, elastin is a polymer composed of cross-linked subunits of a precursor protein, tropoelastin. Elastin-producing cells secrete tropoelastin as an approx. $70 \mathrm{kDa}$ monomer into the extracellular space, where it is rapidly cross-linked to form mature elastin [1]. Ultrastructural analysis of developing elastic fibres demonstrates the presence of 10-12 nm filaments, termed 'microfibrils', in association with elastin [2]. Studies of elastic fibres in fetal development show that early in gestation microfibrils form before the deposition of elastin. With time, microfibrils gradually become covered with insoluble elastin. These observations have led to the hypothesis that microfibrils provide a scaffolding for tropoelastin deposition and serve to align cross-linking domains within tropoelastin monomers for subsequent cross-linking [1,3].

A number of proteins have been identified as components of microfibrils. Among the most abundant and best characterized are the fibrillins and microfibril-associated glycoprotein (MAGP). The fibrillins are large (approx. $350 \mathrm{kDa}$ ) glycoproteins with a common modular arrangement of epidermal growth factor-like repeats and domains with homology with transforming growth factor $\beta-1$ binding protein [4]. MAGP, in contrast, is a small $(31 \mathrm{kDa})$ glycoprotein with post-translational modifications that include O-linked glycosylation and tyrosine sulphation [5,6]. MAGP cannot be extracted from microfibrils in the absence of reducing agent, indicating that disulphide bonds join MAGP to other microfibril components. Recent work also shows that MAGP is a substrate for transglutaminase crosslinking, which may further stabilize microfibril structure [7]. Examination of the amino acid sequence of MAGP suggests the presence of two structural domains [8]. The N-terminal half of MAGP has a net negative charge and is rich in proline, glutamic acid and glutamine residues. Conversely the C-terminal half of MAGP has a positive charge and contains all 13 cysteine residues.

Tropoelastin has a structure consisting predominantly of alternating hydrophobic and cross-linking domains. There are 16 potential cross-linking domains in bovine tropoelastin, all of which contain two or three lysine residues separated by two or three intervening amino acids. Cross-linking occurs when the $\epsilon$-amino group on lysine is oxidized by lysyl oxidase and condenses with a second lysine side chain to form covalent bonds within or between tropoelastin monomers. Based on the spatial and chemical requirements for cross-link formation, it is clear

Abbreviations used: $\mathrm{AT}_{\mathrm{e}}$, antibody to domains $4 / 5$ in elastin; blotto, PBS/0.5\% Tween-20/3\% (v/v) non-fat dry milk; $\mathrm{CT}_{\mathrm{e}}$, antibody to C-terminus of elastin; FBC, fetal bovine auricular chondroblast; FITC, fluorescein isothiocyanate; GAM, goat anti-mouse immunoglobulin; GAPDH, glyceraldehyde phosphate dehydrogenase; GAR, goat anti-rabbit immunoglobulin; HDBSA/NGS, heat-denatured BSA containing normal goat serum; HRP, horseradish peroxidase; MAGP, microfibril-associated glycoprotein; RITC, 3 rhodamine isothiocyanate; SVAS, supravalvular aortic stenosis.

$\S$ To whom correspondence should be addressed. 
that cross-link arrangement is not random. Rather, specific cross-linking domains must be specifically aligned during the polymerization process $[9,10]$. This observation further supports the concept that elastin formation is guided by an organizing structure.

Tropoelastin contains an additional functional domain located at the $\mathrm{C}$-terminus. This region of the protein has a strongly basic net charge and contains the only two cysteine residues found in the molecule. These cysteine residues form an intramolecular disulphide bond, creating a loop and a charged pocket [11]. Recent experiments in vitro, demonstrating binding between tropoelastin and a recombinant form of MAGP, suggest that interaction between the two proteins is mediated by the $\mathrm{C}$ terminal domain of tropoelastin [7,12].

In this study we used domain-specific antibodies directed against tropoelastin and MAGP to define regions of the molecules that interact in elastic fibre formation in vivo. This was done by culturing auricular chondrocytes in the presence of $\mathrm{Fab}$ fragments of these antibodies followed by chemical and morphological examination of elastic fibres formed in the extracellular matrix. We were able to confirm that the C-terminal domain of tropoelastin is responsible for incorporation of tropoelastin monomers into the growing elastic fibre. Our results also suggest that the $\mathrm{N}$ terminal half of MAGP might be responsible for interactions with tropoelastin.

\section{MATERIALS AND METHODS \\ Materials}

ABTS/peroxidase development kit was from Kirkegaard \& Perry Laboratories (Gaithersburg, MD, U.S.A.). Goat anti-mouse (GAM) and goat anti-rabbit (GAR) peroxidase conjugates and nitrocellulose were purchased from Bio-Rad Laboratories (Richmond, CA, U.S.A.). Fluorescein isothiocyanate (FITC)and 3 rhodamine isothiocyanate (RITC)-conjugated goat antimouse and goat anti-rabbit secondary antibodies were obtained from Organon Teknika-Cappel (Durham, NC, U.S.A.). Fourwell Lab-Tek slides were purchased from Nunc (Naperville, IL, U.S.A.). Hybond- $\mathrm{H}^{+}$blotting membrane was from Amersham (Arlington Heights, IL, U.S.A.). DNA Random Prime Labeling Kit was purchased from Gibco-BRL (Gaithersburg, MD, U.S.A.). Pepsin was from Calbiochem (San Diego, CA, U.S.A.). All other chemicals were obtained from Sigma Chemical Co. (St. Louis, MO, U.S.A.).

\section{Cell culture}

Auricular chondrocytes were obtained from the ear cartilage of 180 day gestation bovine fetuses (FBC-180) as described elsewhere [13]. Cells were maintained in Dulbecco's modified Eagle's medium supplemented with $10 \%(\mathrm{v} / \mathrm{v})$ calf serum, non-essential amino acids, L-glutamine and penicillin/streptomycin. All cells were used at first passage. Chondrocytes were seeded at $1 \times 10^{5}$ cells per well in four-well Lab-Tek slides for immunofluorescence. For amino acid analysis and Northern blotting, cells were plated at $3 \times 10^{5}$ cells per well in a six-well plate. Fab fragments of antibodies were added at $0,1,10$ or $100 \mu \mathrm{g} / \mathrm{ml}$ at the time of plating. After 3 days of growth, cultures were fed with fresh medium containing the appropriate Fab. Cells and associated extracellular matrix were analysed after 6 days of treatment.

\section{Antibody and Fab production}

Domain-specific antibodies to the C-terminus of bovine tropoelastin ( $\mathrm{CT}_{\mathrm{e}}$; amino acid residues $\left.741-760\right)$, a sequence encoded by exon 4/5 $\left(\mathrm{AT}_{\mathrm{e}}\right.$; residues 55-72), a sequence near the $\mathrm{N}$ terminus of MAGP (MAGP:21-35), the central portion of MAGP (MAGP:58-70) and the Arg-Gly-Asp (RGD) domain of fibrillin-1 (Fib15d) were prepared from synthetic peptides [7,14]. The antibody to the $\mathrm{C}$-terminal domain of tropoelastin was made against a peptide containing the folded, disulphide-bonded loop structure [11]. The tropoelastin monoclonal BA4 has been previously described [15]. Antibodies were IgG-purified by octanoic acid precipitation [16] and affinity-purified with peptide conjugated to Sepharose.

Fab fragments of antibodies were generated by pepsin digestion and reductive alkylation. Antibodies were dialysed into $70 \mathrm{mM}$ sodium acetate $/ 50 \mathrm{mM} \mathrm{NaCl}, \mathrm{pH} 4.0$, concentrated to $5 \mathrm{mg} / \mathrm{ml}$ and treated with pepsin overnight at $37^{\circ} \mathrm{C}$ at an enzyme-tosubstrate ratio of $1: 33$. After buffer exchange into $0.5 \mathrm{M}$ Tris, $\mathrm{pH} \mathrm{8.3,} \mathrm{the} \mathrm{digest} \mathrm{was} \mathrm{reduced} \mathrm{with} 10 \mathrm{mM}$ 2-mercaptoethanol for $1 \mathrm{~h}$ at room temperature. Iodoacetamide was added to $12 \mathrm{mM}$ and the digest was incubated for $15 \mathrm{~min}$ on ice in the dark. The Fab fragments were then dialysed into $7.5 \mathrm{mM}$ Tris $/ 150 \mathrm{mM} \mathrm{NaCl}, \mathrm{pH} 7.5$, and purified on a $1.5 \mathrm{~cm} \times 90 \mathrm{~cm}$ Sephacryl S-200 column equilibrated in the same buffer.

\section{Indirect immunofluorescence}

FBC-180 cells were grown on four-well Lab-Tek slides as described above. After 6 days of growth, slides were washed twice with PBS and fixed for 20 min with $1 \%$ (w/v) paraformaldehyde prepared in PBS. Cells were then washed twice with PBS and treated with $6 \mathrm{M}$ guanidine hydrochloride/20 mM Tris/

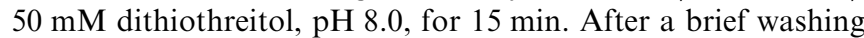
with $20 \mathrm{mM}$ Tris, $\mathrm{pH} 8.0$, the slides were treated with $100 \mathrm{mM}$ iodoacetamide in the dark for $15 \mathrm{~min}$. The cell layers were washed twice with HDBSA/NGS $[1 \mathrm{mg} / \mathrm{ml}$ heat-denatured BSA in PBS with $1 \%(\mathrm{v} / \mathrm{v})$ normal goat serum] and incubated in primary antibody diluted in HDBSA/NGS for $30 \mathrm{~min}$. After three $10 \mathrm{~min}$ washes in HDBSA/NGS, slides were incubated in FITC- or RITC-conjugated secondary antibody diluted $1: 200$ in HDBSA/NGS for $30 \mathrm{~min}$. Slides were washed three times for $30 \mathrm{~min}$ in HDBSA/NGS, and cover slips were mounted with Gelmount containing $1 \mathrm{mg} / \mathrm{ml}$-phenylenediamine. Immunofluorescence was detected with a Nikon Fluophot microscope. BA4 and Fib15d antibodies were used at 1:50 dilution. MAGP:58-70 was diluted $1: 100$. All incubations were performed at room temperature.

\section{Quantification of cross-linking amino acids}

Elastin cross-links in Fab-treated FBC-180 cultures were quantified by amino acid analysis. Chondrocyte cultures were treated as described earlier. After 6 days of growth, medium was removed and the cell layer was rinsed three times for $5 \mathrm{~min}$ at room temperature with PBS $/ 0.5 \%$ Tween-20 to lyse the cells and remove cytosolic proteins. The cell layer was scraped into hydrolysis tubes with $0.5 \mathrm{ml}$ of $6 \mathrm{M} \mathrm{HCl}$. The wells were rinsed with an additional $0.5 \mathrm{ml}$ of $6 \mathrm{M} \mathrm{HCl}$, which was pooled with the first scrapings. Hydrolysis tubes were sealed and heated at $110^{\circ} \mathrm{C}$ overnight. Samples were dried by rotary evaporation, resuspended in sample buffer and analysed on a Beckman model 6300 amino acid analyser with a program modified for resolving the cross-linking amino acids [10].

\section{Northern analysis}

Total RNA was extracted from 6-day-old chondrocyte cultures by guanidine isothiocyanate/phenol extraction. RNA ( $5 \mu \mathrm{g}$ per 

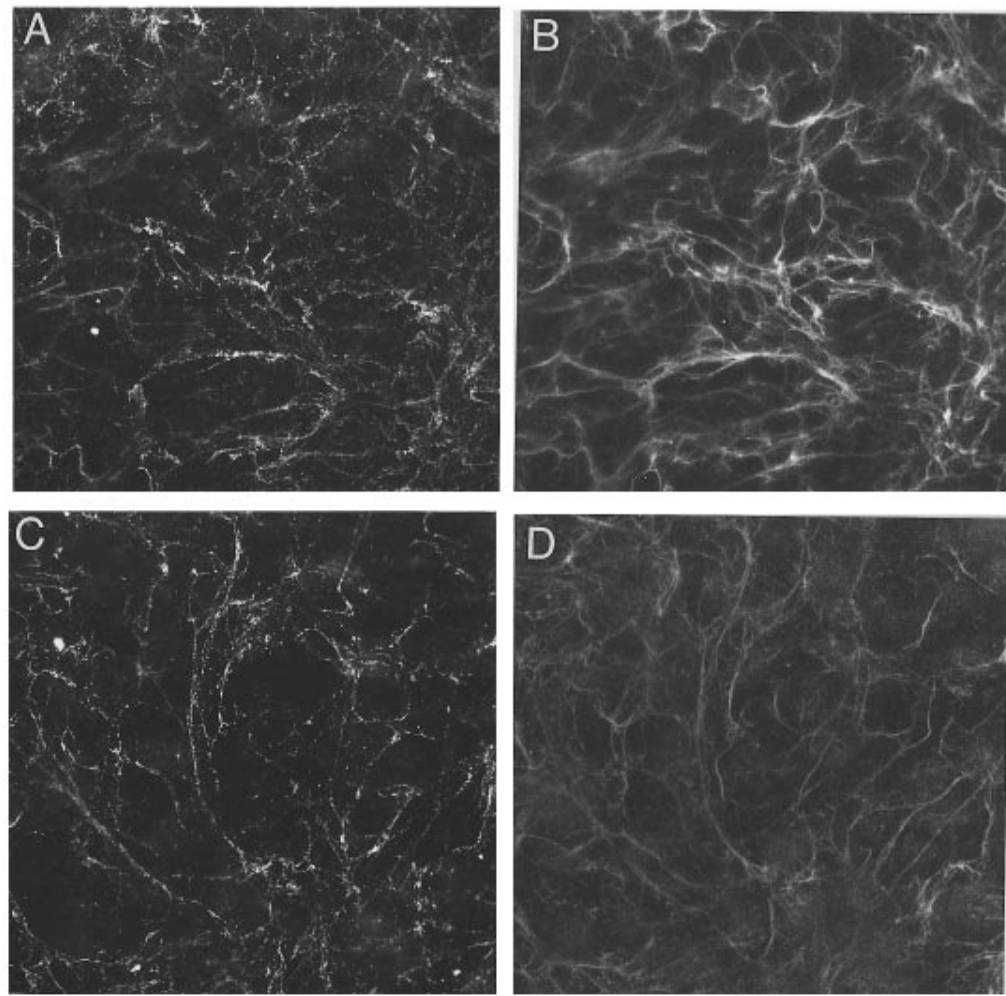

\section{Figure 1 Co-localization of tropoelastin with the microfibril proteins fibrillin-1 and MAGP}

FBC-180 cells were plated at 100000 cells per well in four-well culture slides. After 6 days the cell layers were fixed, reduced and alkylated, and simultaneously stained with antibodies specific for elastin and microfibril components. (A, B) Double-labelling indirect immunofluorescence demonstrating co-localization of elastin (A; monoclonal antibody BA4) and MAGP (B; antibody MAGP:5870). (C, D) Co-localization of elastin (antibody BA4) with fibrillin (antibody Fib-15d) respectively. Magnification $\times 200$.

lane) was subjected to electrophoresis through a $1 \%(\mathrm{w} / \mathrm{v})$ agarose/1 $\mathrm{M}$ formaldehyde gel and then transferred by capillary action to Hybond- $\mathrm{H}^{+}$. Bovine tropoelastin $(1.2 \mathrm{~kb})$ and glyceraldehyde phosphate dehydrogenase (GAPDH) cDNAs were labelled with $\left[\alpha_{-}^{32} \mathrm{P}\right] \mathrm{dCTP}$ and unincorporated isotope was removed by $\mathrm{P} 30$ gel chromatography. Hybridization and washes of the blot were performed as described [17]. Autoradiographic signals were quantified by densitometry and normalized to the GAPDH signal.

\section{Tropoelastin ELISA}

Tropoelastin levels in culture medium of Fab-treated and control chondrocytes were determined by direct-binding ELISA [18]. Vinyl 96-well ELISA plates were coated overnight at $4{ }^{\circ} \mathrm{C}$ with $100 \mu \mathrm{l}$ of conditioned medium. Plate wells were then washed three times with PBS $/ 0.5 \%$ Tween- $20 / 3 \%$ (v/v) non-fat dry milk (blotto) and incubated with the same buffer at $37^{\circ} \mathrm{C}$ for $30 \mathrm{~min}$ to block unreactive binding sites. BA4 antibody diluted to $12 \mu \mathrm{g} / \mathrm{ml}$ in blotto was added to each well and the plate was incubated for $1 \mathrm{~h}$ at $37^{\circ} \mathrm{C}$. After washing three times with blotto, goat anti-rabbit immunoglobulin conjugated to horseradish peroxidase (GAR-HRP) at 1:1000 dilution in blotto was added and the plate was incubated at $37^{\circ} \mathrm{C}$ for $1 \mathrm{~h}$. Wells were washed twice with blotto, followed by a final wash with PBS. Colour was developed with ABTS/peroxidase development kit and was measured at $405 \mathrm{~nm}$.

\section{Western blots}

MAGP and tropoelastin were subjected to SDS/PAGE $[10 \%$ or $12 \%(\mathrm{w} / \mathrm{v}) \mathrm{gel}]$ in the presence of reducing agent. Protein was electrotransferred to nitrocellulose, and non-specific binding sites on the nitrocellulose were blocked by incubation in blotto for $30 \mathrm{~min}$ at room temperature. Fab fragments were diluted to $50 \mu \mathrm{g} / \mathrm{ml}$ in blotto and incubated on the blots overnight at $4{ }^{\circ} \mathrm{C}$. Blots were washed twice for $10 \mathrm{~min}$ with blotto and incubated with GAR-HRP diluted 1:500 in PBS for $2 \mathrm{~h}$ at room temperature. After two $10 \mathrm{~min}$ washes in PBS/0.5\% Tween-20, colour was developed with 4-chloro-1-naphthol.

\section{RESULTS}

\section{Immunostaining of tropoelastin, fibrillin and MAGP}

Immunostaining of elastin in the extracellular matrix of cultured chondrocytes shows a honeycomb network. Close examination of the fibres composing the network reveals a typical 'beads on a string' pattern (Figures 1A and 1C). MAGP and fibrillin labelling exhibit the same arrangement of fibres; however, the beaded pattern is absent. Instead, fibres stained for MAGP and fibrillin-1 are sharply defined thin filaments (Figures 1B and 1D). Simultaneous labelling of tropoelastin and MAGP or fibrillin demonstrates co-localization. These results are consistent with earlier observations at the electron-microscopic level and from protein extraction studies, which show that microfibrils made by 

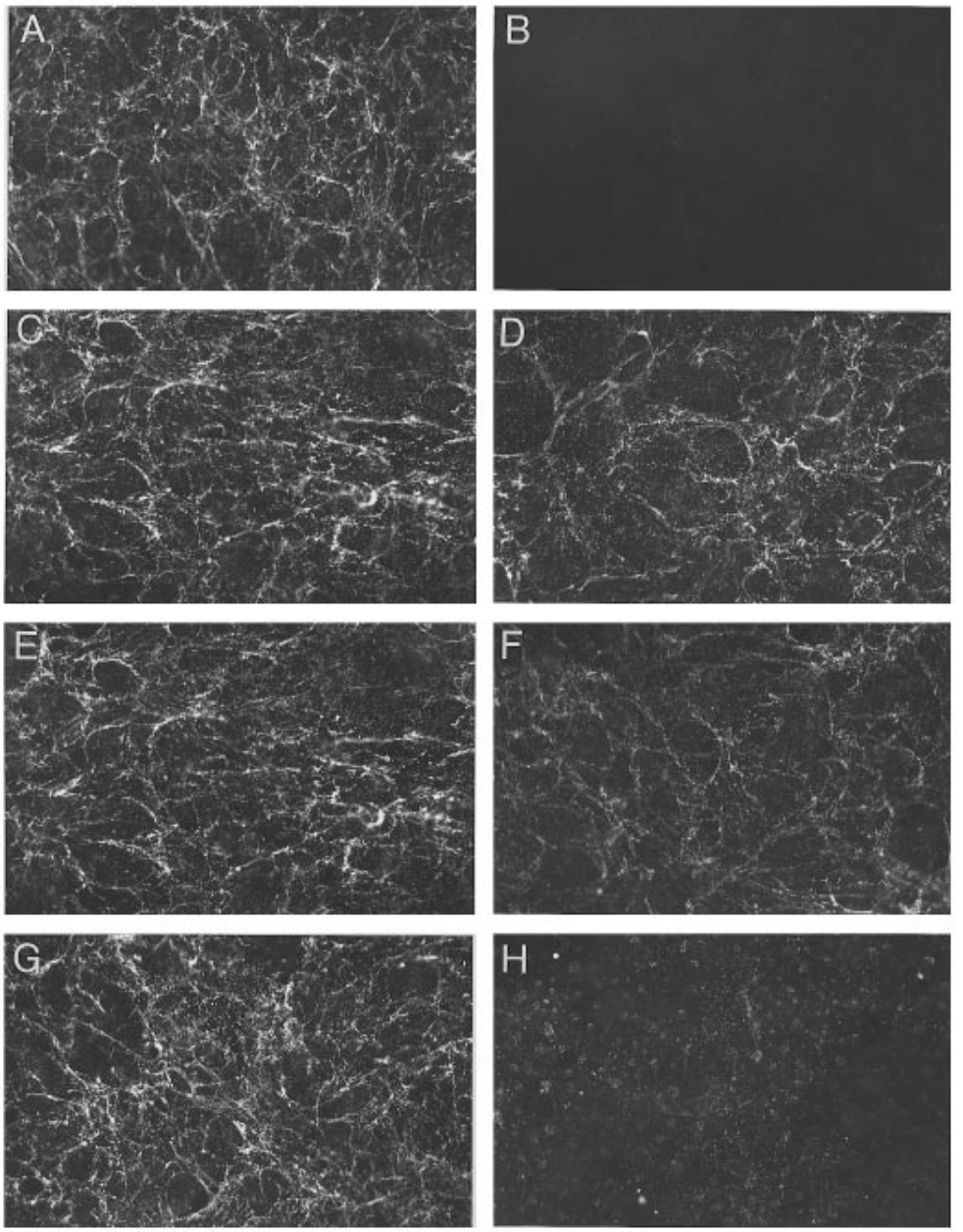

\section{Figure 2 Antibody to the C-terminus of tropoelastin $\left(\mathrm{CT}_{\mathrm{e}}\right)$ blocks elastin deposition into fibres}

First-passage FBC-180 cells were plated in the presence of Fab fragments of antibodies against the C-terminus (CT $)$ or a peptide sequence encoded by exons $4 / 5$ of tropoelastin (AT $)$. Fresh antibody was added after 3 days, and at 6 days elastin was revealed by immunofluorescence microscopy with an elastin monoclonal antibody (BA4) and GAM-FITC. (A) Cells cultured in the absence of Fab fragments. (C, E, G) Elastin staining associated with cells grown in the presence of 1,10 and $100 \mu \mathrm{g} / \mathrm{ml}$ (respectively) of $\mathrm{AT}_{\mathrm{e}}$ Fab. (D, F, H) Elastin staining in the extracellular matrix of cells grown in the presence of $\mathrm{CT}_{\mathrm{e}}$ Fab at 1,10 and $100 \mu \mathrm{g} / \mathrm{ml}$ respectively. (B) Cells treated with $100 \mu \mathrm{g} / \mathrm{ml} \mathrm{AT}_{\mathrm{e}}$ Fab and stained with secondary GAM alone to show that the secondary antibody does not react with the Fab fragment. Magnification $\times 200$.

these cells contain both MAGP and fibrillin-1, and that tropoelastin is tightly associated with microfibrils [19-21].

Solid-phase binding studies with recombinant MAGP and tropoelastin suggest that MAGP is the microfibril component responsible for tropoelastin binding. Further, these studies indicate that this binding occurs through the $\mathrm{C}$-terminal domain of tropoelastin [7,12]. To determine whether these interactions are also important for elastic fibre formation in vivo, we incubated elastin-producing cells with antibodies directed against possible assembly domains on elastin and MAGP. Figure 2 shows the organization of elastic fibres in cultures of FBC-180 cells treated with 1,10 or $100 \mu \mathrm{g} / \mathrm{ml}$ of $\mathrm{CT}_{\mathrm{e}}$. This is compared with FBC-180 cultures treated with the same concentrations of $\mathrm{AT}_{\mathrm{e}}$ (directed against an $\mathrm{N}$-terminal domain in tropoelastin). Elastic fibres in these Fab-treated cultures were revealed with a monoclonal antibody that recognizes a central domain of the molecule. With $1 \mu \mathrm{g} / \mathrm{ml} \mathrm{CT}$, elastin staining was slightly diminished compared with controls. Staining decreased significantly with $10 \mu \mathrm{g} / \mathrm{ml}$ $\mathrm{CT}_{\mathrm{e}}$, and with $100 \mu \mathrm{g} / \mathrm{ml} \mathrm{CT}_{\mathrm{e}}$ organized elastic fibres were sparse. Conversely, elastin staining in cultures treated with $\mathrm{AT}_{\mathrm{e}}$ was unaffected at antibody concentrations of 1,10 and $100 \mu \mathrm{g} / \mathrm{ml}$. $\mathrm{CT}_{\mathrm{e}}$-treated cultures stained with GAM-FITC (Figure 2) or GAR-FITC (Figure 3) in the absence of primary detecting antibody displayed no reactivity and served to demonstrate that the secondary antibodies did not react with the blocking Fab fragments. As shown by the Western blot in Figure 4, both $\mathrm{CT}_{\mathrm{e}}$ and $\mathrm{AT}_{\mathrm{e}}$ retained their reactivity against tropoelastin after conversion into Fab fragments, indicating that the differential ability to block tropoelastin deposition was not due to loss of activity of $\mathrm{AT}_{\mathrm{e}}$. Western blot assays also showed that recognition of tropoelastin by BA4 was not blocked by pretreatment with either $\mathrm{CT}_{\mathrm{e}}$ or $\mathrm{AT}_{\mathrm{e}} \mathrm{Fab}$ fragments (Figure 4). This was an important control to confirm that $\mathrm{CT}_{\mathrm{e}}$ was not merely 'masking' the BA4 antigenic site and thus preventing the detection of elastic fibres by immunofluorescence.

Although $\mathrm{CT}_{\mathrm{e}}$ blocked tropoelastin deposition on to extracellular fibres, it had no effect on the formation of microfibrils as assessed by MAGP and fibrillin-1 staining (Figure 3). At concentrations of $\mathrm{CT}_{\mathrm{e}}$ that produced maximal inhibition of elastin deposition, MAGP and fibrillin-1 staining were comparable with 

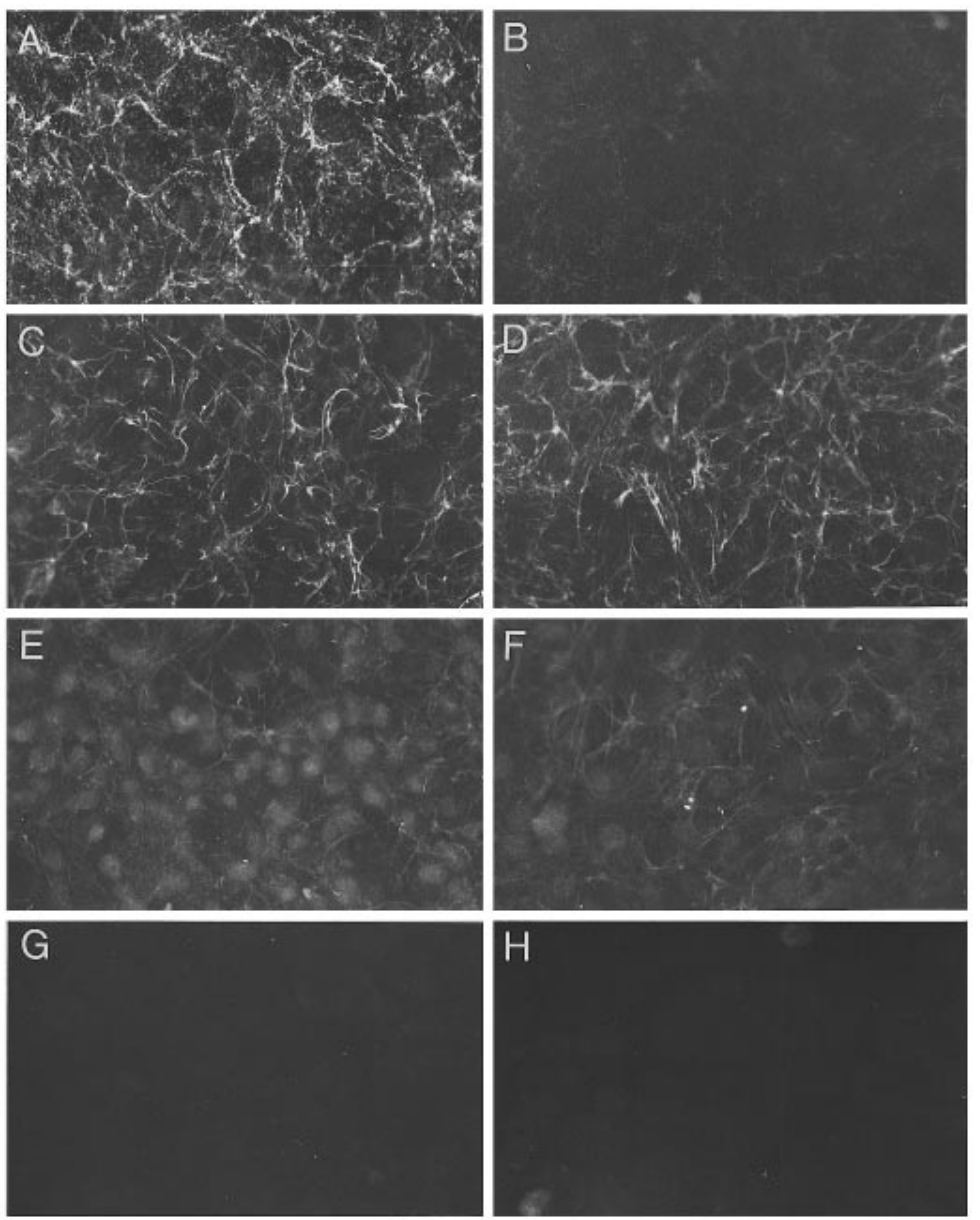

\section{Figure $3 \mathrm{CT}_{\mathrm{e}}$ antibody blocks tropoelastin deposition but has no effect on organization of microfibrils}

FBC-180 cells were cultured as described in Figure 2. (A, C, E, G) Cells grown in the absence of Fab fragments. (B, D, F, H) Cells grown in the presence of $100 \mu \mathrm{g} / \mathrm{ml} \mathrm{CT}$. Fab. (A) and $(\mathbf{B})$ were stained for elastin by using the monoclonal antibody BA4; (C) and (D) were stained for MAGP by using antibody MAGP:58-70; (E) and $(\mathbf{F})$ were stained for fibrillin-1; (G) and $(\mathbf{H})$ show cells stained with GAR-FITC secondary antibody alone. Magnification $\times 200$.

controls. Fibrillin-1 staining was slightly brighter in the $\mathrm{CT}_{\mathrm{e}^{-}}$ treated culture, possibly because the staining antibody had better access to the fibrillin antigenic sites in the absence of accumulated elastin.

Incubation of cells with an antibody to an $\mathrm{N}$-terminal domain of MAGP had a similar effect to that seen with $\mathrm{CT}_{\mathrm{e}}$. As shown in Figure 5, $100 \mu \mathrm{g} / \mathrm{ml}$ MAGP:21-35 blocked elastin deposition on to fibres but had no effect on microfibril formation as assessed by staining with an antibody to a second site in MAGP (MAGP:58-70) or with antibodies to fibrillin-1. Western blot analysis demonstrated that MAGP:21-35 does not cross-react with tropoelastin (results not shown). These results support our data from experiments in vitro suggesting that tropoelastin binds to the MAGP component of microfibrils. Further, these results suggest that the binding site for tropoelastin might be contained in the $\mathrm{N}$-terminal half of MAGP

\section{Elastin secretion and cross-linking}

Tropoelastin monomers are polymerized into insoluble elastin by the formation of cross-links between oxidized lysine residues. A major product of this cross-linking reaction is desmosine, which is unique to elastin and is therefore indicative of the amount of cross-linked elastin deposited in the extracellular matrix. Direct analysis of desmosine levels found in the extracellular matrix of the Fab-treated and control FBC-180 cells confirmed changes in elastin deposition induced by the antibodies. $\mathrm{CT}_{\mathrm{e}}$-treated cells contained the least desmosine (approx. $25 \%$ of control), whereas cells treated with $\mathrm{AT}_{\mathrm{e}}$ were only $20 \%$ below control levels. Together these results support the immunofluorescence data showing decreased matrix-associated elastin in $\mathrm{CT}_{\mathrm{e}}$-treated cultures.

Auricular chondrocytes are efficient at depositing tropoelastin into the extracellular matrix, so that in the absence of inhibitors of cross-linking little soluble tropoelastin can be detected in the medium conditioned by these cells [13]. We were, however, able to detect low levels of tropoelastin in the medium of control FBC-180 cultures by using a direct-binding ELISA assay. In agreement with decreased elastin deposition in the extracellular matrix shown by immunofluorescence and desmosine analysis, an approximate 3 -fold increase in the amount of tropoelastin relative to control was found in the medium of $\mathrm{CT}_{\mathrm{e}}$-treated cultures. Tropoelastin levels were only slightly elevated and not 


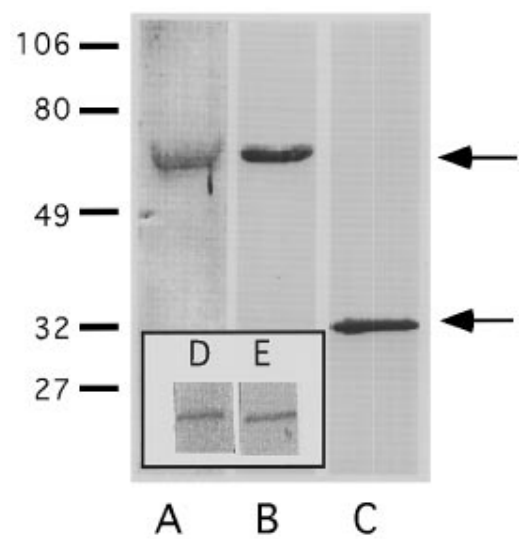

Figure 4 Fab fragments retain reactivity to tropoelastin and MAGP and do not block the binding of BA4 monoclonal antibody to tropoelastin

Western blot of purified tropoelastin (lanes A and B) and MAGP (lane C) developed with $50 \mu \mathrm{g} / \mathrm{ml} \mathrm{Fab}$ fragments of the antibodies $\mathrm{CT}_{\mathrm{e}}$ (lane A), $\mathrm{AT}_{\mathrm{e}}$ (lane B) or MAGP:21-35 (lane C). Fab-specific GAR-HRP secondary antibody and 4-chloro-1-naphthol were used for detection. Lanes $D$ and $E$ (insert) show a blot of tropoelastin incubated with the $\mathrm{CT}_{\mathrm{e}}$ (lane $\mathrm{D}$ ) and $\mathrm{AT}_{\mathrm{e}}$ (lane E) Fab fragments. BA4 monoclonal antibody was then added and detected with a GAM-HRP secondary antibody. The upper arrow indicates the position of tropoelastin; the lower arrow indicates MAGP. The numbers on the left indicate molecular mass markers ( $k D a)$.
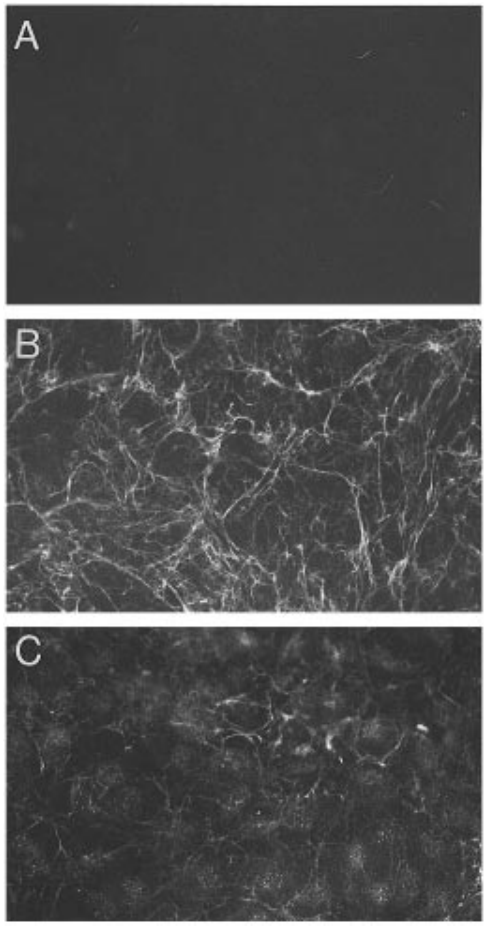

Figure 5 Tropoelastin deposition into fibres is blocked by MAGP:21-35 Fab

FBC-180 cells were grown in the presence of an antibody $(100 \mu \mathrm{g} / \mathrm{ml})$ directed against an Nterminal domain of MAGP (MAGP:21-35). (A) Indirect immunofluorescence staining with a monoclonal antibody to elastin. (B) Staining with an antibody against a central domain of MAGP (MAGP: 58-70). (C) Staining for fibrillin-1. Magnification $\times 175$

significantly above control in cultures treated with $\mathrm{AT}_{\mathrm{e}}$. Tropoelastin mRNA levels determined by Northern blot analysis and normalized to GAPDH were essentially unchanged for the control and antibody-treated cultures, indicating that the antibodies had no effect on tropoelastin gene expression.

\section{DISCUSSION}

Because of their ability to form extracellular elastic fibres in culture, auricular chondrocytes provide a useful model for dissecting the molecular interactions that occur in elastic fibre assembly. We found that we could block deposition and crosslinking of tropoelastin by culturing chondrocytes in the presence of a domain-specific antibody directed against the C-terminus of tropoelastin. This result was demonstrated by the absence of elastic fibre immunofluorescence staining in the matrix, despite normal microfibril staining. We also saw a corresponding decrease in elastin-specific cross-links and an increase in soluble tropoelastin in the medium in $\mathrm{CT}_{\mathrm{e}}$-treated cultures. These results corroborate earlier observations suggesting that the C-terminal domain of tropoelastin is responsible for organization of tropoelastin monomers in the extracellular matrix before cross-linking. A second antibody directed against an $\mathrm{N}$-terminal domain in tropoelastin $\left(\mathrm{AT}_{\mathrm{e}}\right)$ had no effect on tropoelastin deposition, as assayed by elastic fibre immunostaining. Analysis of desmosine levels in the extracellular matrix of $\mathrm{AT}_{\mathrm{e}}$-treated cells indicated a slight decrease in cross-links compared with controls. Because $\mathrm{AT}_{\mathrm{e}}$ is directed against a cross-linking domain at the N-terminus of tropoelastin, it is possible that $\mathrm{AT}_{\mathrm{e}}$ impairs cross-link formation at this one site but has no effect on the insertion of tropoelastin into developing fibres. This explanation is supported by the finding that tropoelastin levels in the media of $\mathrm{AT}_{\mathrm{e}}$-treated cultures are nearly identical with controls, whereas levels in the media of $\mathrm{CT}_{\mathrm{e}}$-treated cultures were elevated, indicating impaired deposition. A similar result was seen by Marigo et al. [22], who found that the formation of amorphous bundles of elastin was inhibited by culturing in the presence monoclonal antibodies to tropoelastin. Deposition of tropoelastin on microfibrils, however, did not seem to be impaired.

Solid-phase binding studies with recombinant MAGP have been used to document binding between MAGP and tropoelastin. We were able to demonstrate that these two proteins interact on intact microfibrils by blocking tropoelastin deposition with an antibody directed against the N-terminus of MAGP (MAGP:21-35). Interestingly, formation of microfibrils was not affected by this antibody, as shown by normal MAGP and fibrillin-1 staining patterns. Because we were unable to generate an antibody specific for the C-terminal domain of MAGP, we could not directly test the hypothesis that the C-terminus is responsible for interaction with other microfibril proteins, or rule out the possibility that the C-terminus of MAGP has some role in binding tropoelastin. On the basis of the clustering of cysteine residues within the C-terminus of MAGP and the necessity for reducing agents for release of MAGP from microfibrils, however, it seems that the C-terminal region of MAGP is responsible for interactions with other microfibril proteins. This, together with our results indicating that tropoelastin binding activity is in the $\mathrm{N}$-terminus, suggests that the two domains in MAGP are functionally distinct.

The tropoelastin gene has recently been linked with an inherited vascular disorder, supravalvular aortic stenosis (SVAS) [23]. SVAS causes narrowing of large elastic arteries, resulting in increased vascular pressure and possible heart failure. Curran et al. [24] reported an autosomal dominant SVAS kindred with a balanced translocation disrupting the elastin gene. Sequencing through the breakpoint region showed that the elastin gene on one allele lacked the C-terminal domain. It has not been determined whether a truncated tropoelastin is secreted by cells 
carrying this mutation, but our results suggest that the absence of the C-terminal domain would prevent incorporation into the elastic fibre. The resulting decrease in tropoelastin deposition could be the source of the pathology seen in SVAS.

In summary, we have demonstrated the use of cultured auricular chondrocytes as a model system for targeted disruption of elastic fibre assembly. By culturing chondrocytes in the presence of Fab fragments of domain-specific antibodies, we have verified that the $\mathrm{CT}$ domain of tropoelastin interacts with microfibrils in the development of elastic fibres. Further, we have shown that the interaction between tropoelastin and microfibrils can be disrupted with an antibody directed against the $\mathrm{N}$ terminus of MAGP. It will be of great interest to determine the exact sequences in MAGP that mediate its interactions with tropoelastin and other microfibrillar proteins.

We thank Clarina Tisdale and Lisa Mecham for technical assistance, and Terese Hall for secretarial support. This work was supported by National Institutes of Health grants HL53325, HL41926 and HL29594 to R.P.M. and AR41474, AR20553 to J.R.

\section{REFERENCES}

1 Mecham, R. P. and Heuser, J. E. (1991) in Cell Biology of Extracellular Matrix, 2nd edn. (Hay, E. D., ed.), pp. 79-109, Plenum Press, New York

2 Low, F. N. (1962) Anat. Rec. 142, 131-137

3 Ross, R., Fialkow, P. J. and Altman, K. (1977) Adv. Exp. Med. Biol. 79, 7-17

4 Maslen, C. L., Corson, G. M., Maddox, B. K., Glanville, R. W. and Sakai, L. Y. (1991) Nature (London) 352, 334-337

5 Gibson, M. A., Hughes, J. L., Fanning, J. C. and Cleary, E. G. (1986) J. Biol. Chem. 261, 11429-11436

6 Tomasini-Johansson, B. R., Ruoslahti, E. and Pierschbacher, M. D. (1993) Matrix 13 203-214

Received 30 November 1995/25 March 1996; accepted 9 April 1996
7 Brown-Augsburger, P., Broekelmann, T., Mecham, L., Mercer, R., Gibson, M. A., Cleary, E. G., Abrams, W. R., Rosenbloom, J. and Mecham, R. P. (1994) J. Biol. Chem. 269, 28443-28449

8 Gibson, M. A., Sandberg, L. B., Grosso, L. E. and Cleary, E. G. (1991) J. Biol. Chem. 266, 7596-7601

9 Gray, W. R., Sandberg, L. B. and Foster, J. A. (1973) Nature (London) 246, 461-466

10 Brown-Augsburger, P., Broekelmann, T., Sloan, C. and Mecham, R. P. (1995) J. Biol. Chem. 270, 17778-17783

11 Brown, P. L., Mecham, L., Tisdale, C. and Mecham, R. P. (1992) Biochem. Biophys. Res. Commun. 186, 549-555

12 Bashir, M. M., Abrams, W. R., Rosenbloom, J., Kucich, U., Bacarra, M., Han, M.-D., Brown-Augsburger, P., Mecham, R. P. and Rosenbloom, J. (1994) Biochemistry 33, 593-600

13 Mecham, R. P. (1987) Methods Enzymol. 144 (D), 232-246

14 Mariencheck, M. C., Davis, E. C., Zhang, H., Ramirez, F., Rosenbloom, J., Gibson, M. A., Parks, W. C. and Mecham, R. P. (1995) Connect. Tissue Res. 31, 87-97

15 Wrenn, D. S., Griffin, G. L., Senior, R. M. and Mecham, R. P. (1986) Biochemistry 25, 5172-5176

16 Harlow, E. and Lane, D. (1988) Antibodies: a Laboratory Manual, Cold Spring Harbor Laboratory Press, Cold Spring Harbor, NY

17 Parks, W. C., Secrist, H., Wu, L. C. and Mecham, R. P. (1988) J. Biol. Chem. 263, 4416-4423

18 Prosser, I. W., Whitehouse, L. A., Parks, W. C., Stahle-Bäckdahl, M., Hinek, A., Park, P. W. and Mecham, R. P. (1991) Connect. Tissue Res. 25, 265-279

19 Gibson, M. A. and Cleary, E. G. (1987) Immunol. Cell Biol. 65, 345-356

20 Kumaratilake, J. S., Gibson, M. A., Fanning, J. C. and Cleary, E. G. (1989) Eur. J. Cell Biol. 50, 117-127

21 Sakai, L. Y., Keene, D. R., Glanville, R. W. and Bachinger, H. P. (1991) J. Biol. Chem. 266, 14763-14770

22 Marigo, V., Daga-Gordini, D., Sitta, A., Volpin, D. and Bressan, G. M. (1992) Eur. J. Cell Biol. 57, 254-264

23 Ewart, A. K., Morris, C. A., Atkinson, D., Jin, W., Sternes, K., Spallone, P., Stock, A. D., Leppert, M. and Keating, M. T. (1993) Nature Genet. 5, 11-16

24 Curran, M. E., Atkinson, D. L., Ewart, A. K., Morris, C. A., Leppert, M. F. and Keating, M. T. (1993) Cell 73, 159-168 